\title{
Comparison of Thessaly Test with Joint Line Tenderness and McMurray Test in the Diagnosis of Meniscal Tears
}

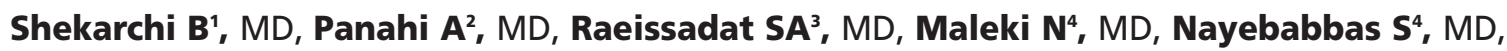 \\ Farhadi $\mathbf{P}^{5}, \mathrm{MD}$ \\ 'Department of Radiology, AJA University of Medical Sciences, Tehran, Iran \\ ${ }^{2}$ Chronic Respiratory Diseases Research Center, National Research Institute of Tuberculosis and Lung Diseases \\ (NRITLD), Tehran, Iran \\ ${ }^{3}$ Department of Physical Medicine and Rehabilitation, Shaheed Beheshti University of Medical Sciences, Tehran, Iran \\ ${ }^{4}$ Department of Physical Medicine and Rehabilitation, AJA University of Medical Sciences, Tehran, Iran \\ ${ }^{5}$ Department of Radiology, Shiraz University of Medical Sciences, Shiraz, Iran
}

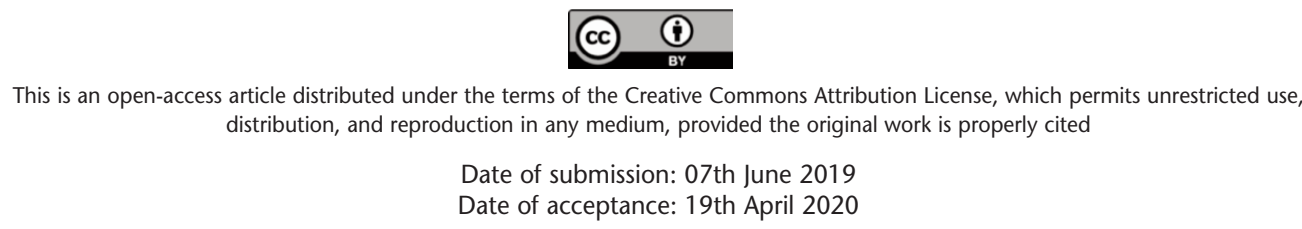

\section{ABSTRACT}

Introduction: Meniscus injuries are the most frequent problem of the knee. The aim of this study was to investigate the accuracy of the Thessaly test and comparing it with those of McMurray and Joint-line tenderness tests for diagnosing meniscal tears.

Materials and methods: This study was designed as a prospective observational one done in an outpatient clinic at a university hospital. 106 patients with knee pain and 82 agematched control were included during study period (from February 2014 to January 2015). Each patient was clinically examined with McMurray, Thessaly, and joint line tenderness tests. Then, the findings were matched by MRI and arthroscopic findings. The sensitivity, specificity, positive predictive value, negative predictive value and accuracy were calculated as main outcomes.

Results: Based on MRI, Thessaly was the most sensitive for medial meniscus tears (56.2\%), while McMurray and jointline tenderness were more specific $(89.1 \%$ and $88.0 \%$, respectively). For lateral meniscus tears, McMurray was the most sensitive $(56.2 \%)$ and all were specific (McMurray $89.6 \%$, Thessaly $88.4 \%$, joint-line tenderness $90.2 \%$ ). With arthroscopy, Thessaly was the most sensitive for medial meniscus (76.6\%), while McMurray and joint-line tenderness were more specific $(81.0 \%$, and $81.0 \%)$. Agreement with arthroscopy was the highest with McMurray (for medial meniscus kappa $=0.40, \mathrm{p}<0.001$, and for lateral meniscus kappa $=0.38, \mathrm{p}=0.002$ ).

Conclusion: The Thessaly can be used to screen for medial meniscus tears. McMurray and joint-line tenderness should be used for suspected medial meniscus tears. For lateral meniscus, McMurray is appropriate for screening and all the tests are useful in clinic.
Keywords:

Thessaly, joint line tenderness, McMurray, meniscal tears

\section{INTRODUCTION}

Knee pain comprises approximately one third of all outpatient physical therapy visits ${ }^{1}$ of which $9 \%$ are associated with meniscal lesions ${ }^{2}$. A combination of subjective symptoms increases the predictive value of recognising meniscal lesion to $70-80 \%$, but patient history is not enough for accurate diagnosis ${ }^{3}$.

Various physical tests have been described in the literature: the McMurray's, the Apley compression and distraction, and the joint line tenderness test with their adaptations are known examples ${ }^{4-14}$. The diagnostic accuracy of the clinical tests may improve with compressive forces applied to the knee joint with weight-bearing axial compression ${ }^{15,16}$. Medial joint line tenderness, knee locking, daily pain and work absence have been reported to predict $61 \%$ of meniscal tear confirmed by arthroscopy ${ }^{3}$.

More recently, the Thessaly test was suggested to be safely used as a first line screening test for identifying the candidates of arthroscopic meniscal surgery. The test also was reported to have a lower false positive and false negative compared to other clinical tests ${ }^{16}$. On the other hand, MRI is considered as the diagnostic method of choice because of providing a reliable non-invasive tool in identifying common knee pathology $y^{4,17}$. Arthroscopy is also the gold standard for the diagnosis of meniscal lesions, with $90-95 \%$ accuracy ${ }^{18}$. In addition, arthroscopy provides simultaneous therapeutic possibilities. 
Low specificity, sensitivity, and diagnostic accuracy of the clinical tests may leave meniscal lesions undetected. The methodological quality of the studies on the clinical tests has a significant effect on reported sensitivities and specificities as well ${ }^{2,19}$.

High cost and false positives are the disadvantages of MRI for the diagnosis of meniscal lesions ${ }^{4,20}$. A false positive of $65 \%$ for identifying medial meniscal tears and $43 \%$ for lateral meniscus tears have been estimated for MRI when compared with surgical findings ${ }^{21}$. Even, some researchers believe that MRI is not superior to physical examination in the diagnosis of meniscal tears ${ }^{20}$. Invasiveness and high cost are the major drawbacks for common use of arthroscopy too ${ }^{4}$.

Therefore, we need less expensive, non-invasive, and more accurate methods of identifying meniscal lesions. The optimal clinical tests and measures should be selected with high sensitivity and specificity. The aim of conducting the present study was to investigate the accuracy of the Thessaly test in screening for meniscal tears. We examined whether the Thessaly test alone or in combination with other clinical tests could provide a non-invasive means of identifying lesions.

\section{MATERIALS AND METHODS}

The present study was designed as a prospective observational one which was conducted from February 2014 to January 2015 in an outpatient clinic of physical medicine and rehabilitation at the university hospital; Imam Reza, a large referral practice and research centre in Tehran. The study protocol was approved by the institutional review board (IRB) of AJA University of Medical Sciences and we obtained ethics approval from the local ethics committee before the study was commenced. All the participants read and signed an informed consent form prior to study involvement.

Consecutive patients aged more than 15 years who had a current knee pain or discomfort, history of swelling or effusion, knee locking, or clicking sensation on movement of the knee were enrolled in the study. Exclusion criteria included individuals with a previous history of knee surgery or arthroscopy, and the radiologic evidence or clinical manifestations of osteoarthritis and rheumatologic diseases. In addition, we excluded patients if they could not attend the clinical examinations and did not take MRI or refused arthroscopy.

At the first visit and according to predetermined inclusion and exclusion criteria patients were screened for eligibility. From 220 eligible patients, 106 completed the tests and composed our analytic sample. We also selected 100 age- matched participants as the group control from which 82 completed the study. The control group was randomly selected from patients who came to the clinic for complaints other than knee problems (e.g., pain in their shoulders).

At the beginning of the study, research staff was briefed. Our clinical assessors were two specialists of physical medicine and rehabilitation. The study was carried out under the supervision of two board-certified physical medicine and rehabilitation specialists, and an associate professor of radiology.

Patients were interviewed to obtain their past medical histories. The presence of the symptoms and signs of meniscal lesions and other knee problems were questioned. In addition, the recruitment questionnaire asked about consultation with a physician and active or previous treatment and surgery. Then our clinical assessors performed physical examinations and conducted further investigations for the diagnosis of meniscal lesions. We recorded any pain or discomfort, clicking sensation or locking on movement, and swelling and injury of the knee.

For the Thessaly test, participants were instructed to stand while supported by holding their hands. Thereafter, they were guided to perform internal and external rotation of the knee and body in three occasions during which the knee was flexed at almost $20^{\circ}$. We considered the test as positive when the patients had medial or lateral joint-line discomfort or experienced locking in the knee ${ }^{4}$. For all the participants, we performed McMurray and joint line tenderness at the lateral and medial sides. Further investigations including MRI were ordered and lesions were graded ${ }^{22}$. Then, the patients with suspected meniscal lesions were referred to arthroscopy for definitive diagnosis and appropriate treatment.

We used a block design to randomise the patients to the observers. The investigators who performed physical examinations were blinded to patients' medical history. Moreover, the assigned radiologists also were unaware of the study question and patients' medical history.

All statistical analyses were performed with the Statistical Package for Social Sciences version 16.0 [SPSS Inc, Chicago, IL, USA]. The distribution of variables was analysed with Kolmogorov-Smirnov test. Continuous data are presented as mean and standard deviation, and categorical data, as numbers and proportions. We used Chisquared and t-test to assess differences in demographic characteristics between the two groups. Sensitivity, specificity, positive and negative predictive values, positive and negative likelihood ratios, the accuracy of diagnoses, and diagnostic odds were calculated by forming two-by-two cross table and using standard formula. Combinations of the tests were also evaluated statistically to find the best 
diagnostic strategy in the clinic. A p-value of less than 0.05 was considered significant.

\section{RESULTS}

A total of 188 patients were included in the study. The mean age of the patients in was found to be $23.9 \pm 5.1$ (range 19-44) years, and sex distribution was 115 males (61.2\%) and 73 females $(38.8 \%)$. We performed MRI for all patients. Overall, $96(51 \%)$ participants had medial meniscus tears including $23(12.2 \%)$ grade I, $42(22.3 \%)$ grade II, 27 (14.4\%) grade III, and 4 (2.1\%) grade IV. Also, 16 (8.5\%) patients had lateral meniscus tears including $7(3.7 \%)$ grade I, $3(1.6 \%)$ grade II, and $6(3.2 \%)$ grade III. There was no grade IV lateral meniscus tear among the participants. Other findings on MRI included 63 (33.5\%) incomplete and 16 (8.5\%) complete anterior cruciate, $2(1.1 \%)$ incomplete posterior cruciate, $36(19.1 \%)$ incomplete medial and lateral collateral, $9(4.8 \%)$ incomplete lateral collateral, and 1 complete lateral collateral ligament tears. In total, 126 (67\%) participants had at least one lesion in their menisci or cruciate ligaments, confirmed by MRI. Of these 126 patients, 26 were from the group control. In addition, 7 participants had combined tears of menisci and anterior cruciate ligament. Based on MRI, Thessaly was the most sensitive for medial meniscus tears (56.2\%), while McMurray and jointline tenderness were more specific $(89.1 \%$ and $88.0 \%$, respectively). For lateral meniscus tears, McMurray was the most sensitive (56.2\%) and all were specific (McMurray
$89.6 \%$, Thessaly $88.4 \%$, joint-line tenderness $90.2 \%$ ). Table I, demonstrates the diagnostic parameters based on the results of MRI.

As the gold standard, arthroscopy was performed for 68 (36.2\%) patients and the findings included 47 (69\%) medial and $16(23 \%)$ lateral meniscus tears. In addition, we found $40(59 \%)$ anterior and $1(1.5 \%)$ posterior cruciate ligament tears on arthroscopy. Thessaly was the most sensitive for medial meniscus tears $(76.6 \%)$, while McMurray and jointline tenderness were more specific $(81.0 \%$, and $81.0 \%)$. The most sensitive test for lateral meniscus tears was combined tests with $68.8 \%$ sensitivity, while McMurray test had highest specificity (83\%). (Table II) demonstrates the diagnostic parameters based on the results of knee arthroscopy. We also measured the agreements between the results of the clinical tests and arthroscopy for the diagnosis of meniscal tears (Table III).

\section{DISCUSSION}

Meniscal tears are the result of injury or degeneration of the substance of the meniscus. Majority patients with meniscal tears complain of an acute onset of sharp pain following a twisting injury with the knee flexed and the foot planted on the ground ${ }^{23}$. The diagnosis of meniscus tear can frequently be made from a careful history, physical examination and appropriate diagnostic tests. There are various provocative manoeuvres or tests to elicit symptoms from a torn meniscus,

Table I: The diagnostic parameters of the three clinical tests with MRI as the standard method of diagnosis $(\mathrm{N}=188)$

\begin{tabular}{|c|c|c|}
\hline \multirow[b]{2}{*}{ Clinical test } & \multicolumn{2}{|c|}{ Diagnosis } \\
\hline & Medial meniscus tear & Lateral meniscus tear \\
\hline \multicolumn{3}{|l|}{ McMurray } \\
\hline Sensitivity & $44.8 \%$ & $56.2 \%$ \\
\hline Specificity & $89.1 \%$ & $89.6 \%$ \\
\hline False positive & 10 & 17 \\
\hline False negative & 53 & 7 \\
\hline Accuracy & $66.4 \%$ & $83.0 \%$ \\
\hline \multicolumn{3}{|c|}{ Joint-line tenderness } \\
\hline Sensitivity & $43.8 \%$ & $43.8 \%$ \\
\hline Specificity & $88.0 \%$ & $90.2 \%$ \\
\hline False positive & 11 & 16 \\
\hline False negative & 54 & 9 \\
\hline Accuracy & $65.4 \%$ & $82.4 \%$ \\
\hline \multicolumn{3}{|l|}{ Thessaly } \\
\hline Sensitivity & $56.2 \%$ & $50.0 \%$ \\
\hline Specificity & $79.3 \%$ & $88.4 \%$ \\
\hline False positive & 19 & 19 \\
\hline False negative & 42 & 8 \\
\hline Accuracy & $67.5 \%$ & $81.4 \%$ \\
\hline \multicolumn{3}{|c|}{ All the tests combined } \\
\hline Sensitivity & $66.7 \%$ & $68.8 \%$ \\
\hline Specificity & $73.9 \%$ & $84.3 \%$ \\
\hline False positive & 24 & 27 \\
\hline False negative & 32 & 5 \\
\hline Accuracy & $70.0 \%$ & $83.0 \%$ \\
\hline
\end{tabular}


Table II: The diagnostic parameters of the three clinical tests with arthroscopy as the gold standard of the diagnosis $(\mathrm{N}=68)$

\begin{tabular}{|c|c|c|}
\hline \multirow[b]{2}{*}{ Clinical test } & \multicolumn{2}{|c|}{ Diagnosis } \\
\hline & Medial meniscus tear & Lateral meniscus tear \\
\hline \multicolumn{3}{|l|}{ McMurray } \\
\hline Sensitivity & $66.0 \%$ & $56.2 \%$ \\
\hline Specificity & $81.0 \%$ & $83.0 \%$ \\
\hline False positive & 4 & 8 \\
\hline False negative & 16 & 7 \\
\hline Accuracy & $70.5 \%$ & $70.5 \%$ \\
\hline Positive predictive value & $88.6 \%$ & $59.2 \%$ \\
\hline Negative predictive value & $51.5 \%$ & $84.8 \%$ \\
\hline Positive likelihood ratio & 3.5 & 3.3 \\
\hline Negative likelihood ratio & 0.4 & 0.5 \\
\hline \multicolumn{3}{|l|}{ Joint-line tenderness } \\
\hline Sensitivity & $61.7 \%$ & $50.0 \%$ \\
\hline Specificity & $81.0 \%$ & $78.7 \%$ \\
\hline False positive & 4 & 10 \\
\hline False negative & 18 & 8 \\
\hline Accuracy & $67.6 \%$ & $66.2 \%$ \\
\hline Positive predictive value & $87.9 \%$ & $44.4 \%$ \\
\hline Negative predictive value & $48.6 \%$ & $82.2 \%$ \\
\hline Positive likelihood ratio & 3.2 & 2.3 \\
\hline Negative likelihood ratio & 0.5 & 0.6 \\
\hline \multicolumn{3}{|l|}{ Thessaly } \\
\hline Sensitivity & $76.6 \%$ & $50.0 \%$ \\
\hline Specificity & $52.4 \%$ & $74.5 \%$ \\
\hline False positive & 10 & 12 \\
\hline False negative & 11 & 8 \\
\hline Accuracy & $69.0 \%$ & $63.2 \%$ \\
\hline Positive predictive value & $78.3 \%$ & $40.0 \%$ \\
\hline Negative predictive value & $50.0 \%$ & $81.4 \%$ \\
\hline Positive likelihood ratio & 1.6 & 2.0 \\
\hline Negative likelihood ratio & 0.4 & 0.7 \\
\hline \multicolumn{3}{|l|}{ All the tests combined } \\
\hline Sensitivity & $83.0 \%$ & $68.8 \%$ \\
\hline Specificity & $42.9 \%$ & $69.2 \%$ \\
\hline False positive & 12 & 16 \\
\hline False negative & 8 & 5 \\
\hline Accuracy & 70.5 & $69.0 \%$ \\
\hline Positive predictive value & $76.5 \%$ & $40.7 \%$ \\
\hline Negative predictive value & $52.9 \%$ & $87.8 \%$ \\
\hline Positive likelihood ratio & 1.4 & 2.2 \\
\hline Negative likelihood ratio & 0.4 & 0.4 \\
\hline
\end{tabular}

Table III: Agreements between the results of the clinical tests and arthroscopy

\begin{tabular}{|lcccc|}
\hline & Clinical tests & Proportion in agreement & Kappa & p-value \\
\hline Medial meniscus & McMurray & $70.6 \%$ & 0.40 & $<0.001$ \\
& Joint-line Tenderness & $67.6 \%$ & 0.36 & 0.001 \\
\multirow{3}{*}{ Lateral meniscus } & Thessaly & $69.1 \%$ & 0.29 & 0.018 \\
& McMurray & $76.2 \%$ & 0.38 & 0.002 \\
\multirow{2}{*}{ Both menisci } & Joint-line Tenderness & $71.4 \%$ & 0.28 & 0.028 \\
& Thessaly & $68.3 \%$ & 0.23 & $0.069^{*}$ \\
& All the tests combined & $81.0 \%$ & 0.16 & $0.105^{*}$ \\
\hline
\end{tabular}

* Non-significant 
but the reported accuracy have been inconsistent across studies. In this study, we tried to compare the accuracy of Thessaly test with those of McMurray and joint line tenderness in screening for meniscal tears and to examine whether the tests alone or combined could provide a noninvasive means of identifying lesions. We compared the results of the clinical tests with findings of MRI as a noninvasive method, and of arthroscopy as the diagnostic gold standard.

Our results demonstrated that when MRI is the basis for the diagnosis of medial meniscus tears, Thessaly is the most sensitive, while McMurray and joint-line tenderness are more specific clinical tests. In other words, a negative Thessaly test is more helpful in ruling out medial meniscus tears and a positive McMurray or joint-line tenderness test can be used for ruling in the tears. For lateral meniscus tears McMurray is the most sensitive and all the three tests are highly specific.

With arthroscopy the results again showed that for the diagnosis of medial meniscus tears Thessaly is the most sensitive while McMurray and joint-line tenderness are more specific clinical tests. The three tests seem to be equally accurate for the diagnosis of medial meniscus tears. Positive predictive value is the largest for McMurray, while negative predictive value is almost the same for the three tests. In addition, McMurray test possesses the highest positive likelihood ratio. For lateral meniscus tears McMurray is the most sensitive, specific, and accurate test with the largest positive predictive value and likelihood ratio. For tears in anterior cruciate ligament, medial and lateral menisci, together, joint-line tenderness is the most sensitive and McMurray is the most specific. Agreement between the result of the clinical tests and arthroscopy was the highest for McMurray test.

In a study by Gupta et $a l^{24}$ with 66 knees to validate joint line tenderness, the sensitivity, specificity, positive predictive value, negative predictive value and accuracy in diagnosing medial meniscus tears found to be $50 \%, 61.7 \%, 51.8 \%, 60 \%$ and $56.4 \%$, respectively. The sensitivity, specificity, positive predictive value, negative predictive value and accuracy for McMurray's test for diagnosing medial meniscus tear were $54 \%, 79 \%, 68 \%, 67.50 \%$ and $67.74 \%$, respectively. The author concluded that clinical tests like McMurray and joint line tenderness have low diagnostic value when applied individually. They may be useful when combined together with the background of clinical history. The diagnostic parameters of McMurray test was in concordance with our results, while those were lower than our study regarding joint line tenderness.

In a study by Karachalios et $a l^{16}, 213$ patients with knee injuries underwent MRI and arthroscopic surgery, and 197 asymptomatic participants underwent MRI on their normal knees. It was reported that the Thessaly test at $20^{\circ}$ of knee flexion had a diagnostic accuracy of $94 \%$ for the detection of medial meniscus tears and $96 \%$ for the lateral meniscus tears, and that the test had a low rate of false-positive and false-negative recordings. They suggested that other clinical tests showed inferior accuracies, except for joint line tenderness with the accuracy of $89 \%$ in the detection of lateral meniscal tears. It was concluded that the Thessaly test at $20^{\circ}$ of knee flexion can be used effectively as a first-line clinical screening test. They even claimed that the test will reduce the need for and the cost of modern MRI methods. Our results failed to show the superiority of the Thessaly test. In another study on 109 (80 males) patients with average age of 39 (16 to 56) years, the diagnostic accuracy of the Thessaly test was compared with joint line tenderness and McMurray's tests. Patients were presented with a history suggestive of a meniscal tear. They underwent MRI and arthroscopy. The study showed a relatively low diagnostic accuracy for the Thessaly (61\% for medial meniscus and $80 \%$ for lateral meniscus), and the McMurray's tests $(57 \%$ for medial meniscus and $77 \%$ for lateral meniscus). It was declared that the joint line tenderness test is the most accurate ( $81 \%$ for medial meniscus and $90 \%$ for lateral meniscus). Also, it was suggested that combining the tests further increased the accuracy. It was concluded that the Thessaly test in isolation was not useful for the detection of meniscal tears but it helps to increase diagnostic certainty when combined with other tests ${ }^{4}$. With regard to the Thessaly test and when MRI was considered as the standard diagnostic method, the results of that study were comparable to ours. However, we calculated similar accuracies for both menisci, when arthroscopy was used as the gold standard. Other similarities can be recognised between the two studies for the McMurray test, especially when MRI is considered as the basis for the comparisons. But, our results did not support the concept of superiority for the joint line tenderness test, specifically for arthroscopy as the gold standard. It should be noted that there are other discrepancies between the two studies. For example, we selected participants randomly, and used a control group to provide higher methodological quality.

Our sample was sufficiently large to detect important differences among the diagnostic parameters. Our research team and assessors were highly trained, and they attempted to follow diagnostic protocols strictly. Meanwhile, ethical considerations prevent us to perform arthroscopy when it was not necessary. Therefore, we were not able to compare the clinical tests with the gold standard for all participants.

\section{CONCLUSION}

Our study showed that the Thessaly is a sensitive clinical test for the diagnosis of medial meniscus tears. Therefore, the test can be used to screen general population. On the other hand, McMurray and joint-line tenderness are more specific 
and should be used to diagnose medial meniscus tears in patients with the related clinical manifestations. For lateral meniscus tears, the McMurray test is appropriate for screening and all the three tests are useful in the physical examination of patients with the suspected tears. Therefore, with regard to sensitivity, specificity, and accuracy, the Thessaly should be used along with the other tests. Overall, we did not find important difference in the diagnostic usefulness of the three tests and prefer to use the tests combined. The tests combined have a high proportion in agreement with arthroscopy and are valuable in the detection of meniscal and the cruciate ligament lesions.

\section{ETHICAL CONSIDERATION}

Ethics approval was obtained from the institutional review boards, and the study protocol was carried out in accordance with the Declaration of Helsinki. The rationale of the study was explained to all participants. Patients were informed that they were free to withdraw from the study at any time. A trained study nurse accompanied patients, and provided patients with verbal information if needed, and a leaflet on the diagnostic procedures to eligible participants. All patients gave written informed consent.

\section{SOURCE OF FUNDING}

The authors declare that they did not receive any financial support from any public or private sources.

\section{CONFLICT OF INTEREST}

The authors declare that they have no competing interests.

\section{REFERENCES}

1. Jette AM, Delitto A. Physical therapy treatment choices for musculoskeletal impairments. Phys Ther. 1997; 77(2): 145-54. doi: $10.1093 / \mathrm{ptj} / 77.2 .145$

2. Meserve BB, Cleland JA, Boucher TR. A meta-analysis examining clinical test utilities for accessing meniscal injury. Clin Rehabil. 2008; 22(2): 143-61. doi: 10. 1177/0269215507080130

3. Abdon P, Lindstrand A, Thorngren KG. Statistical evaluation of the diagnostic criteria for meniscal tears. Int Orthop. 1990;14(4):341-5. doi: 10.1007/BF00182641

4. Konan S, Rayan F, Haddad FS. Do physical diagnostic tests accurately detect meniscal tears?. Knee Surg Sports Traumatol Arthrosc. 2009; 17(7): 806-11. doi: 10. 1007/s00167-009-0803-3

5. Eren OT. The accuracy of joint line tenderness by physical examination in the diagnosis of meniscal tears. Arthroscopy. 2003; 19(8): 850-4. doi: 10.1016/s0749-8063(03)00736-9

6. Jackson JL, O'Malley PG, Kroenke K. Evaluation of acute knee in pain in primary care. Ann Intern Med. 2003; 139 (7): 575-88. doi: 10.7326/0003-4819-139-7-200310070-00010.

7. Grover M. Evaluating acutely injured patient for internal derangement of the knee. Am Fam Physician. 2012; 85(3): 47-52.

8. Ercin E, Kaya I, Sungur I, Demirbas E, Ugras AA, Cetinus EM. History, clinical findings, magnetic resonance imaging, and arthroscopic correlation in meniscal lesions. Knee Surg Sports Traumatol Arthrosc. 2012; 20(5): 851-6. doi: 10. 1007/s00167011-1636-4

9. Jain DK, Amaravati R, Sharma G. Evaluation of the clinical signs of anterior cruciate ligament and meniscal injuries. Indian J Orthop. 2009; 43(4): 375-8. doi: 10.4103/0019-5413.55466

10. Wadey VMR, Mohtadi NGH, Bray RC, Frank CB. Positive predictive value of maximal posterior joint-line tenderness in diagnosing meniscal pathology: a pilot study. Can J Surg. 2007; 50(2): 96-100.

11. Sae-Jung S, Jirarattanaphochai K, Benjasil T. KKU knee compression-rotation test for detection of meniscal tears: a comparative study of its diagnostic accuracy with McMurray test. J Med Assoc Thai. 2007; 90(4): 718-23.

12. Rose RE. The accuracy of joint line tenderness in the diagnosis of meniscal tears. West Indian Med J. 2006; 55(5): 323-6. doi: 10.1590/s0043-31442006000500006

13. Pookarnjanamorakot C, Korsantirat T, Woratanarat P. Meniscal lesions in the anterior cruciate insufficient knee: the accuracy of clinical evaluation. J Med Assoc Thai. 2004; 87(6): 618-23. 
14. Malanga GA, Andrus S, Nadler SF, McLean J. Physical examination of the knee: a review of the original test description and scientific validity of common orthopaedic test. Arch Phys Med Rehabil. 2003; 84(4): 592-603. doi: 10.1053/apmr.2003.50026

15. Akseki D, Ozcan O, Boya H, Pinar H. A new weight-bearing meniscal test and a comparison with McMurray's test and joint line tenderness. Arthroscopy. 2004; 20(9): 951-8. doi: 10.1016/j.arthro.2004.08.020

16. Karachalios T, Hantes M, Zibis AH, Zachos V, Karantanas AH, Malizos KN. Diagnostic accuracy of a new clinical test (the Thessaly test) for early detection of meniscal tears. J Bone Joint Surg Am. 2005; 87(5): 955-62. doi: 10.2106/JBJS.D.02338

17. Muellner T, Weinstabl R, Schabus R, Vécsei V, Kainberger F. The diagnosis of meniscal tears in athletes. A comparison of clinical and magnetic resonance imaging investigations. Am J Sports Med. 1997; 25(1): 7-12. doi: 10.1177/036354659702500103

18. Salesnick FH, Noble HB, Bachman DC, Steinberg FL. Internal derangement of the knee: diagnosis by arthrography, arthroscopy, and arthrotomy. Clin Orthop Relat Res. 1985; (198): 26-30.

19. Scholten RJ, Deville WL, Opstelten W, Bijl D, van der Plas CG, Bouter LM. The accuracy of physical diagnostic test for assessing meniscal lesions of the knee: a meta-analysis. J Fam Pract. 2001; 50(11): 938-44.

20. Miller GK. A prospective study comparing the accuracy of the clinical diagnosis of meniscus tear with magnetic resonance imaging and its effect on clinical outcome. Arthroscopy. 1996; 12(4): 406-13. doi: 10.1016/s0749-8063(96)90033-X

21. Ben-Galim P, Steinberg EL, Amir H, Ash N, Dekel S, Arbel R. Accuracy of magnetic resonance imaging of the knee and unjustified surgery. Clin Orthop Relat Res. 2006; 447: 100-4. doi: 10.1097/01.blo.0000203471.50808.b7

22. Hantes ME, Kotsovolos ES, Mastrokalos DS, Ammenwerth J, Paessler HH. Arthroscopic meniscal repair with an absorbable screw: results and surgical technique. Knee Surg Sports Traumatol Arthrosc. 2005; 13(4): 273-9. doi: 10.1007/s00167-004-0527-3

23. Daniel D, Daniels E, Aronson D. The diagnosis of meniscus pathology. Clin Orthop Relat Res. 1982; (163): 218-24.

24. Gupta Y, Mahara D, Lamichhane A. McMurray's Test and Joint Line Tenderness for Medial Meniscus Tear: Are They Accurate?. Ethiop J Health Sci. 2016; 26(6): 567-72. doi: 10.4314/ejhs.v26i6.10 